\title{
Regulatory immune cells in regulation of intestinal inflammatory response to microbiota
}

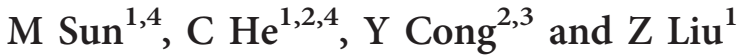

The intestinal lumen harbors nearly 100 trillion commensal bacteria that exert crucial function for health. An elaborate balance between immune responses and tolerance to intestinal microbiota is required to maintain intestinal homeostasis. This process depends on diverse regulatory mechanisms, including both innate and adaptive immunity. Dysregulation of the homeostasis between intestinal immune systems and microbiota has been shown to be associated with the development of inflammatory bowel diseases (IBD) in genetically susceptible populations. In this review, we discuss the recent progress reported in studies of distinct types of regulatory immune cells in the gut, including intestinal intraepithelial lymphocytes, Foxp ${ }^{+}$regulatory T cells, regulatory B cells, alternatively activated macrophages, dendritic cells, and innate lymphoid cells, and how dysfunction of this immune regulatory system contributes to intestinal diseases such as IBD. Moreover, we discuss the manipulation of these regulatory immune cells as a potential therapeutic method for management of intestinal inflammatory disorders.

\section{INTRODUCTION}

The gastrointestinal tract, considered as the largest immune interface with the environment, is continuously exposed to multiple antigens from diets, commensal bacteria, and pathogens. With such a high antigen load, a balance is required between inflammatory responses to harmful pathogens and tolerance to commensal flora or food antigens in maintaining immune homeostasis in the healthy gut. ${ }^{1}$ Both innate and adaptive immunity have been suggested in maintaining intestinal homeostasis and various factors are involved in this process. The breakdown of this well-controlled balance results in intestinal disorders, such as inflammatory bowel diseases (IBD), food allergy, infectious diseases, diarrhea, and even cancer.

IBD comprises two distinct diseases, Crohn's disease (CD) and ulcerative colitis, and is a chronic relapsing disorder of the intestines caused by an exaggerated immune response to intestinal commensal bacteria in genetically susceptible individuals. However, the lesions and symptoms of IBD are disparate. $\mathrm{CD}$ can spread throughout the entire gastrointestinal tract and, thus, patients with CD may present with abdominal pain, diarrhea, weight loss, and fever, whereas ulcerative colitis only affects the colon, with symptoms of rectal bleeding, frequent stool, tenesmus, and lower abdominal pain. Moreover, IBD patients have a high risk of developing extraintestinal autoimmunity (e.g., arthritis, psoriasis, and primary sclerosing cholangitis). ${ }^{2}$ To date, the etiology and pathology of IBD have not been fully elucidated, and multiple factors are considered to play roles in the pathogenesis, including interactions among genetic factors, commensal microbiota, and host immune system. ${ }^{3}$ IBD is characterized by the dysregulated immune responses to microbiota in intestinal mucosa, especially by $\mathrm{CD} 4{ }^{+} \mathrm{T}$ cell-mediated immune responses. For many years, $\mathrm{T}$ helper type 1 (Th1) cells, together with their proinflammatory cytokines (e.g., interferon- $\gamma($ IFN- $\gamma$ ) and tumor necrosis factor (TNF)), were thought to be responsible for CD pathogenesis, whereas ulcerative colitis was considered to be associated with Th2-like responses, characterized by an enhanced production of interleukin (IL)-5 and IL-13. ${ }^{4}$ However, studies on the IL-23/ Th17 axis have demonstrated that a subtype of $\mathrm{CD} 4^{+} \mathrm{T}$ cells that produce IL-17, called Th17 cells, is an essential player in the pathogenesis of IBD. ${ }^{5}$ Recent discoveries have shown that ROR $\gamma \mathrm{t}$ (retinoic acid-related orphan nuclear receptor $\gamma \mathrm{t}$ )dependent innate lymphoid cells (ILCs) are another dominant

\footnotetext{
${ }^{1}$ Department of Gastroenterology, Institute for Intestinal Diseases, Shanghai Tenth People's Hospital, Tongji University, Shanghai, China. ${ }^{2}$ Department of Microbiology and Immunology, University of Texas Medical Branch, Galveston, Texas, USA and ${ }^{3}$ Department of Pathology, University of Texas Medical Branch, Galveston, Texas, USA. Correspondence: Y Cong (yicong@utmb.edu) or Z Liu (liuzhanju88@126.com)

${ }^{4}$ The first two authors contributed equally to this work.
} 


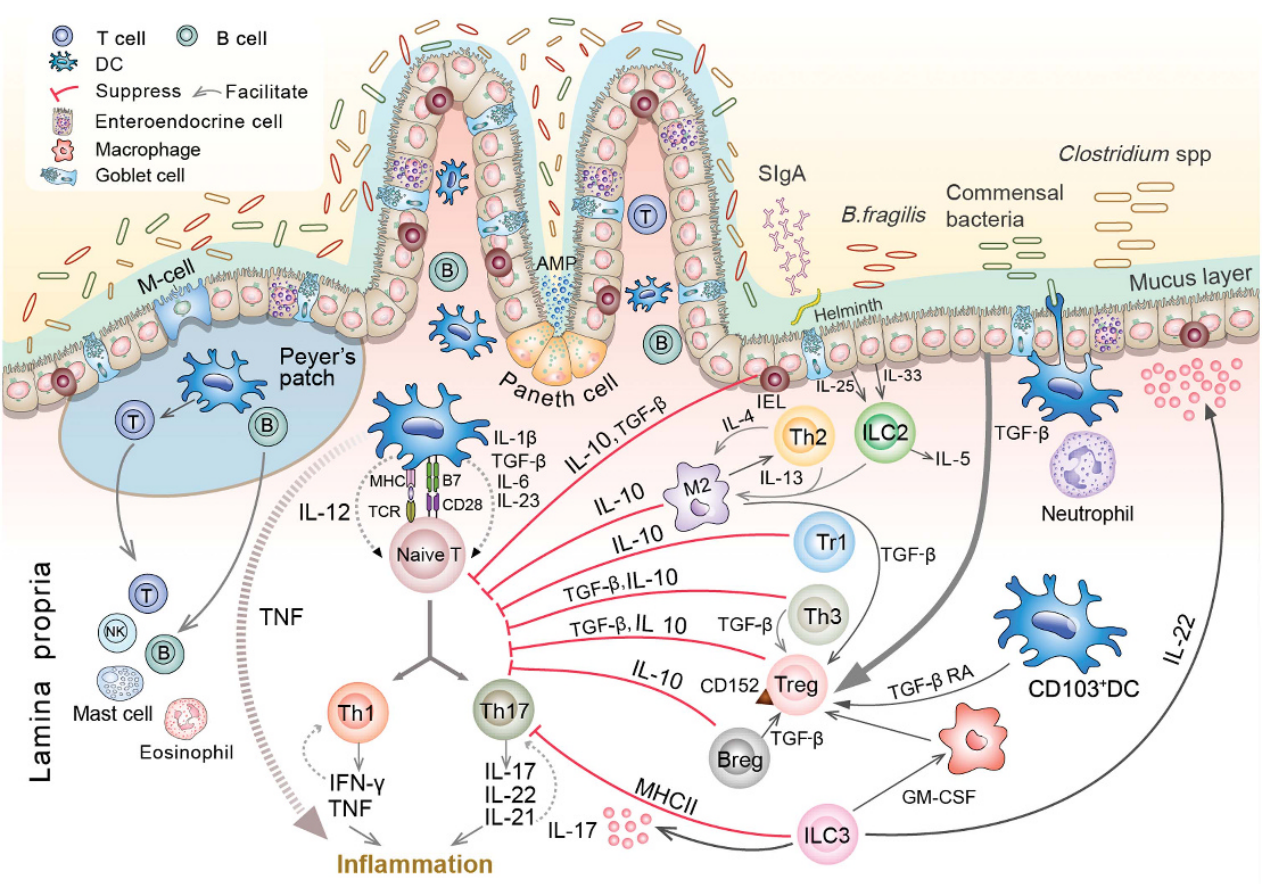

Figure 1 Microbiota and immune regulatory cells in gut. Schematic shows the location of immune regulatory cells and other immune cells and their signaling cascades in the lamina propria of gut. The luminal microbiome lies adjacent to the intestinal epithelium. The microbiota plays an important role in the maintenance of mucosa homeostasis. Breach of the tolerance leads to the development of inflammatory bowel diseases (IBD), inflammation, and tumor. Intestinal epithelial barrier is the first line of defense to prevent the pathogens invasion, including the epithelial tight junction complexes, mucus layer secretion by goblet cell, secretory immunoglobulin A (slgA), antimicrobial protein (AMP) secretion by plasma cells, and Paneth cells. With regard to the innate immune response in intestine, neutrophils could be earlier recruited to inflammatory sites to clear the pathogens. In addition, macrophages, dendritic cells (DCs), and innate lymphoid cells (ILCs) could sense antigens and then secrete cytokines or chemokines to regulate the inflammatory responses. Some innate immune regulatory cells such as M2 induced by interleukin (IL)-4 and IL-13 could directly inhibit effector T cells through IL-10 secretion and promote regulatory T cell (Treg) suppressive function by producing transforming growth factor- $\beta$ (TGF- $\beta$ ). CD103 ${ }^{+}$DCs could regulate intestinal inflammation via facilitating Treg differentiation in a TGF- $\beta$ - and retinoic acid (RA)-dependent manner. ILC3 serve as an antigen-presenting cell (APC) that uniquely restrain the $\mathrm{CD} 4{ }^{+} \mathrm{T}$-cell response in a major histocompatibility complex (MHC) II-dependent manner, and could secrete IL-22 that participates in the repair of impaired epithelium. In response to epithelium-derived IL-25 and IL-33, ILC2 could produce IL-5 and IL-13 to play the role of anti-helminth. The primarily regulatory cells are Tregs that suppress effector T-cell differentiation and proliferation by producing IL-10 and TGF- $\beta$ or expressing CD152. The IL-10-producing type 1 regulatory T (Tr1) cells perform the anti-inflammatory function. Moreover, T helper type 3 (Th3) cells, regulatory B cells (Bregs), and intraepithelial lymphocytes (IELs) have the capacity of suppressing the excessive Th1 and Th17 responses through IL-10 and TGF- $\beta$ production. Therefore, these immune regulatory cells collectively maintain the intestinal homeostasis by interacting with each other.

source of IL-17 that perform both protective and pathogenic roles in the regulation of intestinal mucosal inflammatory responses. ${ }^{6}$ Moreover, accumulating evidences have also suggested that the dysfunction of regulatory immune cells that have the capacity of suppressing excessive inflammatory responses in intestinal mucosa contribute to the induction of $\mathrm{IBD}^{7-10}$

In this review, we will discuss different regulatory cell subsets of the innate and adaptive immune systems, such as intestinal intraepithelial lymphocytes, $\mathrm{T}$ and $\mathrm{B}$ cells, macrophages, dendritic cells (DCs) and ILCs, with a focus on their roles in regulating the immune response that are critical for the maintenance of homeostasis in the gut (Figure 1).

\section{EPITHELIAL BARRIER}

The luminal surface of the human intestine, $\sim 200-400 \mathrm{~m}^{2}$, is an environment where massive microbiota and pathogens directly contact the gut mucosa (Figure 1). Thus, as the first line of defense against invasion of resident microbiota, the intestinal epithelium forms a physical barrier through a tight-junction complex, mucus, antimicrobial proteins, and immunoglobulin A (IgA). Under physiologic conditions, intestinal epithelial cells (IECs) can express apical junction complexes, such as E-cadherin, ZO-1, claudin, and occludin, to form a tight monolayer that could prevent bacteria penetrating the lamina propria. Moreover, IECs provide an immune defense function by secreting IgA and antimicrobial proteins. Indeed, IgA is a key molecule to limit microbiota adherence and access to the intestinal epithelial surface. Previous work has demonstrated that microbiota-specific Th17 cells could promote the secretion of IgA via IL-17dependent intestinal polymeric Ig receptor expression, IL-21dependent $\operatorname{IgA}^{+}$cell differentiation, and IgA class switch recombination. ${ }^{11,12}$ Therefore, downregulation of IgA in IL-17 receptor-deficient mice increases bacterial stimulation and severity of colitis. ${ }^{11}$

In the stimulation of bacteria or helminth, IECs can provide an immune sentinel function by secreting various cytokines, 
including IL-1 family (e.g., IL-1 $\beta$, IL-18, IL-33, IL-37), IL-6, IL-8, and some anti-inflammatory cytokines (e.g., IL-10, transforming growth factor- $\beta$ (TGF- $\beta$ ), IL-25). Studies have shown that IL-1 family members possess pleiotropic functions in different phase of inflammation. IL-1 $\beta$ participates in the inflammatory responses by augmenting infiltration of neutrophils, and by activating T cells and ILCs. ${ }^{13}$ IL-18, also known as IFN- $\gamma$-inducing factor, induces the production of proinflammatory cytokines and chemokines, ${ }^{14}$ and may also exert a role in suppressing colitis and contributing to wound healing. ${ }^{15}$ IL-33 induces Th2 response and drives production of IL-5 and IL-13 by Th2 cells and ILC2. In the chronic dextran sulfate sodium (DSS)-induced colitis model, IL-33 promotes the recruitment of neutrophils for the bacterial clearance ${ }^{16}$ and induces regulatory T-cell differentiation in the TGF- $\beta$ mediated pathway. ${ }^{17}$ IL-37, a novel cytokine that is found to suppress innate immune response, could protect mice from colitis through decreasing the production of IL- $1 \beta$ and TNF and inducing expression of IL-10. ${ }^{18}$ IL-8, a chemokine that stimulates neutrophil migration, could recruit neutrophils from the circulation to inflammatory sites to clear the pathogens. IEC-derived IL- 6 is capable of mediating an inflammatory response through binding to the IL-6R $\alpha$ and gp 130 molecules of target cells, such as T cells. IL-6 in synergy with TGF- $\beta$ can induce ROR $\gamma \mathrm{t}$ expression and Th17 cell differentiation. However, in the presence of IL-6, TGF- $\beta$-induced Foxp3 expression is inhibited. ${ }^{19}$ Evidences have demonstrated that IL- $6^{-1-}$ mice exhibit a mild DSS-induced colitis, ${ }^{20}$ and that blockage of gp130 signaling in IECs pronouncedly decreases the recruitment of neutrophils and macrophages to inflamed sites via reducing the production of chemokines or adhesion molecules and inhibiting the invading ability of myeloid cells. ${ }^{21}$ Furthermore, IL-6 is also a crucial cytokine that could promote the differentiation of B cells and the secretion of IgA. ${ }^{22} \mathrm{IL}-25$ is considered to promote $\mathrm{Th} 2 \mathrm{immune}$ response, and the reduced expression of IL-25 in inflamed mucosa of IBD patients exacerbates intestinal inflammation with the cause that IL-25 could inhibit $\mathrm{CD}^{+}{ }^{+}$T-cell activation. ${ }^{23}$

Besides the IECs, there are other specialized cells including goblet cells that can secrete a mucus inner layer to resist bacterial invasion, and Paneth cells that can secrete various antimicrobial proteins to hamper access and survival of bacteria adjacent to the epithelium. The function of mucus in inhibiting bacterial breach was emphasized by studies demonstrating that MUC2 $^{-1-}$ mice have no bacteria-free zone and can develop intestinal inflammation spontaneously. ${ }^{24}$ Moreover, recent study has also shown that mice with the nuclear factor-кBinducible protein A20 conditional knockout in IECs and myeloid cells (A20 ${ }^{\mathrm{IEC}-\mathrm{KO}}$ mice) could develop severe colitis because of impaired goblet cells causing reduced mucus. ${ }^{25}$ The IECs of $\mathrm{A} 20^{\mathrm{IEC}-\mathrm{KO}}$ mice were found to be sensitive to TNF-induced apoptosis. Thus, A20 possesses a dominant anti-apoptotic effect on IECs, although it could negatively regulate nuclear factor-кB signaling that also plays a role in protecting barrier integrity via inhibiting the apoptosis of IECs. ${ }^{26}$

\section{Intraepithelial lymphocytes}

Beneath the intraepithelial lymphocytes (IECs), that is, in the basal layer of IECs, there is a population of dispersed immune cells called IELs. IELs, the exclusive $\mathrm{T}$ cells that become in direct contact with the enterocytes, are found to play an essential role in the maintenance of intestinal homeostasis.

IELs are specific $\mathrm{T}$ cells that consist of antigen-experienced $\mathrm{T}$-cell subsets bearing T-cell receptor- $\gamma \delta(\mathrm{TCR} \gamma \delta)$ and TCR $\alpha \beta$, and can be divided into two populations, including natural and induced IELs. Natural IELs that have undergone alterative selection with self-antigens and have differentiated into doublenegative $\mathrm{TCR} \gamma \delta^{+}$or $\mathrm{TCR} \alpha \beta^{+}$cells from triple-positive $\left(\mathrm{CD} 4{ }^{+} \mathrm{CD} 8 \alpha \beta^{+} \mathrm{CD} 8 \alpha \alpha^{+}\right)$cells in thymus directly migrate to the intestinal epithelium. ${ }^{27}$ On the other hand, induced IELs that have gone through the conventional selection in thymus and differentiated into naive $\mathrm{CD} 4^{+}$or $\mathrm{CD} 8 \alpha \beta^{+} \mathrm{T}$ cells are activated at mesenteric lymph nodes or Peyer's patches when they accept major histocompatibility complex (MHC) class I peptides and MHC class II peptides, respectively, and then migrate to the intestinal epithelia. The induced IELs include $\mathrm{CD} 4^{+}, \mathrm{CD} 8 \alpha \beta^{+}$, and $\mathrm{CD} 8 \alpha^{+} \beta^{-} \mathrm{TCR} \alpha \beta^{+}$ cells. ${ }^{27}$ Furthermore, CD $8 \alpha \alpha$ homodimer, a hallmark of T-cell activation, is expressed on the majority of IELs and mainly function as a TCR corepressor. ${ }^{28}$ Because of the specific location of IELs in intestinal mucosa, they are involved in resisting to the invasion of foreign pathogens and preserving immune regulation. In response to infection, TCR $\gamma \delta^{+}$IELs can secrete IFN- $\gamma$ and TNF to defend the intestine against pathogens, and importantly to drive proinflammatory effects. ${ }^{29}$ Fascinatingly, challenging the conventional view that TCR $\gamma \delta^{+}$ IELs are stationary in intestinal epithelium, Edelblum et al. ${ }^{30}$ have found that TCR $\gamma \delta^{+}$IELs could migrate within the epithelia and into lamina propria dynamically through occludin-occludin interactions between IECs and TCR $\gamma \delta^{+}$ IELs in the sites of TCR $\gamma \delta^{+}$IEL/IEC contact. Recently, they also found that TCR $\gamma \delta$-deficient mice that were infected with Toxoplasma gondii had upregulated parasite translocation into the lamina propria in comparison with wild-type mice, suggesting that the migration of TCR $\gamma \delta^{+}$IELs into the lateral intercellular space plays a critical role in host defense response to infection. ${ }^{31}$ Therefore, the migration of TCR $\gamma \delta{ }^{+}$IELs exerts a key role in innate immune surveillance and could prevent the translocation of bacteria and pathogens in intestinal epithelia. For intestinal barrier protection, TCR $\gamma \delta^{+}$natural IELs could secrete keratinocyte growth factor-1 to repair the impaired intestinal epithelium. ${ }^{32}$ Studies demonstrated that TCR $\gamma \delta^{+}$ cell-deficient mice suffered from even more serious DSSinduced colitis because of keratinocyte growth factor deficiencies causing tissue repair delay. ${ }^{33}$ In addition, TCR $\gamma \delta^{+} \mathrm{T}$ cell-deficient mice showed an increase in susceptibility to DSSinduced colitis because of reduced mucin expression and impaired goblet cell function. ${ }^{34}$ For immune regulation of intestinal mucosa, $\mathrm{TCR} \gamma \delta^{+} \mathrm{T}$ cells could increase the production of TGF- $\beta$ and IL-10 to perform their suppressive function in intestinal inflammation. ${ }^{35,36} \mathrm{CD} 8 \alpha \alpha^{+} \mathrm{TCR} \alpha \beta^{+}$ IELs have also been reported that provide the protective effects 
for intestinal mucosa by expressing some immune regulatory molecules, such as TGF- $\beta$, lymphocyte activation gene 3 , and killer cell immunoglobulin-like receptors. ${ }^{37}$ In addition, there is evidence that another subset of IELs, CD $8 \alpha^{+} \beta^{-}$TCR $\alpha \beta^{+}$ cells, could prevent inflammation in a naive $\mathrm{CD} 4{ }^{+} \mathrm{CD} 45 \mathrm{RB} \mathrm{B}^{\text {high }}$ $\mathrm{T}$-cell transfer model of colitis in an IL-10-dependent manner. ${ }^{38}$

Collectively, IELs are a critical part of the intestinal immune system, simultaneously participating in the innate and adaptive immune regulation.

\section{REGULATORY T CELLS}

Tregs are a subset of $\mathrm{CD} 4^{+} \mathrm{T}$ cells that play an important role in maintaining peripheral tolerance and are involved in the pathogenesis of various clinical disorders. In the gut, regulatory T cells (Tregs) have also been observed to be a primary mediator in maintaining the immune homeostasis. Conventionally, Tregs are known to be generated in the thymus through IL-2 signaling, referred to as thymus-derived Tregs. However, another defined subset of Tregs has been discovered to develop from Foxp $3^{-} \mathrm{CD} 25^{-} \mathrm{CD} 4{ }^{+}$cells in peripheral organs/tissues in the presence of TGF- $\beta$ and all-trans retinoic acid, and are identified as peripherally derived Tregs. ${ }^{39}$ The surface marker to define Tregs was long unknown until researchers identified the high-affinity IL-2R $\alpha$ chain (CD25) that constitutively expresses in Tregs. However, CD25 was not found to be a unique marker for Tregs, because it is also expressed in activated $\mathrm{T}$ cells. The finding of a forkhead box P3 (Foxp3) transcription factor, which is specifically expressed in CD4 ${ }^{+}$ $\mathrm{CD} 25^{+}$Tregs, provides a unique marker for Tregs. Foxp 3 is not only the marker to define the Treg lineage, but also the key regulatory gene for the development and function of Tregs. ${ }^{40,41}$ The level of Foxp3 protein expression in Tregs is critical for their suppressive function, and deficiency of the Foxp3 gene leads to autoimmune diseases, e.g., polyendocrinopathy, enteropathy, and X-linked autoimmunity allergic dysregulation syndrome. ${ }^{42}$ Several studies have also suggested that when $\mathrm{CD} 4{ }^{+} \mathrm{CD} 25^{-} \mathrm{T}$ cells were transfected with Foxp3 gene, they would possess the behavior like Tregs. Foxp3-transduced T cells have been shown to display suppressive activities and inhibit the development of IBD and autoimmune gastritis in severe combined immunodeficient (SCID) mice. ${ }^{41}$ Consistent with these notions, in the presence of TGF- $\beta$, nonregulatory T cells can be induced to express Foxp3, endowing these cells with the capacity of suppression. ${ }^{43}$

In intestinal lamina propria, Tregs maintain intestine homeostasis through negative regulation of effector $\mathrm{T}$ cells and play a critical role in suppressing intestinal inflammation by producing IL-10 or TGF- $\beta$ and highly expressing cytotoxic T lymphocyte antigen (CTLA-4 or CD152) (Figure 1). ${ }^{44,45}$ However, the abnormal frequency and function of Tregs have been suggested in the pathogenesis of IBD. ${ }^{46-48}$ Tregs have also been reported to remarkably increase in peripheral blood from inactive IBD patients, but decrease in active disease, ${ }^{46}$ and the suppressive role of Tregs in autologous $\mathrm{CD} 25^{-} \mathrm{CD} 4{ }^{+}$T-cell proliferation is impaired in active IBD, ${ }^{48}$ consistent with other discoveries about impaired Treg function in autoimmune conditions such as rheumatoid arthritis. ${ }^{47}$ However, it has also been observed that Tregs from both peripheral blood and mucosal lymphoid tissues of IBD patients still retain suppressive activities. ${ }^{46}$ Thus, whether the suppressive roles of Tregs are impaired in IBD is still elusive.

Several lines of evidence have shown that the altered cytokine milieus of the intestine contribute to the altered frequency and function of Tregs in IBD. Moreover, an imbalance of Tregs and Th17 exists in IBD. In an environment rich in TGF- $\beta$, CD $4^{+}$ $\mathrm{CD} 25^{+}$Foxp $3^{+}$Tregs could not only induce $\mathrm{CD} 4{ }^{+} \mathrm{CD} 25^{-} \mathrm{T}$ cells into IL-17-producing cells, but also facilitate themselves into Th17 cells. A recent study has also demonstrated that IL$17^{+} \mathrm{Foxp}^{+} \mathrm{CD}^{+} \mathrm{T}$ cells are present in the intestinal mucosa of IBD patients. ${ }^{48} \mathrm{CD}$ patients had a markedly higher frequency of $\mathrm{IL}-17^{+} \mathrm{Foxp}^{+} \mathrm{T}$ cells in inflamed intestinal mucosa and circulating blood compared with the levels in healthy controls. Moreover, $\mathrm{CD} 25^{+}$regulatory T cells from IBD patients have been demonstrated to have an impaired suppressive function compared with healthy controls that is associated with the coexpression of ROR $\gamma \mathrm{t}$ and Foxp3 in IL- $17^{+} \mathrm{Foxp} 3^{+} \mathrm{T}$ cells. Our recent work has also demonstrated that psychological stress-derived prolactin is able to induce DCs to secrete IL-6 and IL-23 and facilitate Tregs to differentiate into Foxp3 ${ }^{+}$ $\mathrm{TNF}^{+} \mathrm{IL}-17^{+} \mathrm{T}$ cells, further aggravating intestinal inflammation. In addition, this study highlights the influence of a cytokine environment on immune regulation in gut mucosa. ${ }^{49}$

IBD are associated with upregulation of TNF and, accordingly, anti-TNF therapy is now considered an effective therapy for IBD. Accumulating evidence indicates that anti-TNF therapy has an effect on Tregs. The frequency of peripheral blood Tregs in IBD patients was increased after anti-TNF therapy, and this treatment was able to enhance the number of Tregs in the peripheral blood from chronic IBD cases by reducing the apoptosis of these cells. ${ }^{50}$ In addition, in vitro experiments have indicated that the suppressive role of Tregs in regulating effector $\mathrm{T}$ cells was enhanced after an anti-TNF injection, and Tregs from IBD patients after anti-TNF therapy also displayed an enhanced ability to suppress effector $\mathrm{CD} 4^{+} \mathrm{T}$ cells in vivo. ${ }^{51}$

With respect to the role of bacteria, the commensal bacteria play a crucial role in the maintenance of gut homeostasis and, moreover, abnormal bacteria composition is considered to be one of the pathogenic factors in IBD. Intestinal microbiota regulate the development of the immune system and play an critical role in the induction of Tregs. ${ }^{52}$ Bacteroides fragilis and Clostridia species have been found to suppress an inflammatory response in the intestine by promoting Tregs, and the strain of Clostridium butyricum is also found to induce IL-10-producing macrophage in intestine and prevent DSS-induced acute colitis in mice, ${ }^{53}$ whereas segmented filamentous bacteria could enhance the Th17 response to protect the intestines from infection (e.g., Citrobacter rodentium) in mice. ${ }^{54}$ Multiple mechanisms have been found to be involved in microbiota regulation of Treg differentiation and function. For example, polysaccharide A of $B$. fragilis could promote the suppressive 
activity of inducible Foxp $3^{+}$Tregs via Toll-like receptor 2 signaling and protect the TNBS (2,4,6-trinitrobenzenesulfonic acid)-induced colitis in mice. ${ }^{55}$ Different bacterial species could regulate the cytokine milieu to affect $\mathrm{T}$-cell differentiation and activation. IL-6, IL-21, IL-23, and IL-27 could prevent Treg development, but facilitate the induction of proinflammatory Th1/Th17 cells. ${ }^{56,57}$ Of note, the same commensal bacteria in a different context are also able to induce varying immune responses. B. fragilis, unbalanced Clostridia flora, and altered Schaedler flora species tend to exert a suppressive function by inducing Tregs in normal conditions of the intestine, whereas they could enhance Th1/Th17 cell development under the context of an absence of Tregs. ${ }^{54,58}$ Furthermore, recent work has also indicated that the bacterial metabolites are involved in regulating the gut immune response. Butyrate, a colon microbial fermentation product, could induce the differentiation of Tregs in the colonic lamina propria and ameliorate $\mathrm{T}$ cell-dependent experimental colitis in mice. ${ }^{59}$ Butyrate could also downregulate the production of proinflammatory mediators (e.g., IL-6, IL-12, nitric oxide) of lamina propria macrophages via inhibiting histone deacetylases, furthering the importance of butyrate in maintenance of tolerance to intestinal microbiota. ${ }^{60}$ In addition, previous work has demonstrated that butyrate could impart anti-inflammatory properties on colonic macrophages and DCs to induce differentiation of Tregs and IL-10-producing $\mathrm{T}$ cells through activation of Gpr109a signaling. ${ }^{61}$

In addition to Foxp ${ }^{+}$Tregs, other distinct sets of Tregs have also been discovered to be involved in immune regulation in gut mucosa. The type 1 regulatory $\mathrm{T}(\mathrm{Tr} 1)$ cells, induced by the presence of IL-10, are a different class of $\mathrm{CD} 4{ }^{+}$Tregs from that of Foxp $3^{+}$Tregs. Tr1 cells display their suppressive function via the production of IL-10 and TGF- $\beta$, whereas the lack of Foxp3 expression is observed in this population. ${ }^{62}$ Previous work has demonstrated that the IL-10-producing Tr1 cells could suppress the antigen-specific proliferation of naive $\mathrm{CD}^{+}{ }^{+} \mathrm{T}$ cells and cotransfer of $\mathrm{Tr} 1 \mathrm{~T}$-cell clones could prevent experimental colitis in SCID mice induced by naive $\mathrm{CD} 4{ }^{+} \mathrm{CD} 45 \mathrm{RB}^{\text {high }}$ splenic $\mathrm{T}$ cells or microbiota antigen-specific Th1 cells. ${ }^{63,64}$ As an anti-inflammatory cytokine, IL-10 is able to directly inhibit cytokine production (e.g., TNF), to indirectly suppress the proliferative responses of antigen-specific $\mathrm{CD} 4{ }^{+} \mathrm{T}$ effector cells, and maintain the persistence of Foxp3 expression in Tregs. ${ }^{65}$ In addition, IL-10 can limit inflammation by indirectly suppressing the antigen-presenting ability of distinct types of professional antigen-presenting cells (APCs). Recently, a phase I/IIa clinical trial has been performed, in which the efficacy and safety of ovalbumin-specific $\operatorname{Tr} 1$ cells were assessed in the treatment of patients with refractory $\mathrm{CD}$, showing that injections of these cells significantly reduced the Crohn's Disease Activity Index of CD patients. ${ }^{66}$

Th3 cells are a unique subset of $\mathrm{CD} 4^{+}$regulatory cells, by which TGF- $\beta$ is required for their biologic activity and regulatory functions, and which are determined during the course of investigating mechanisms of oral tolerance. Like Tr1 and Foxp $3^{+}$Tregs, Th 3 cells secrete a large amount of TGF- $\beta$, low level of IL-4 and IL-10, but no IFN- $\gamma$ or IL-2. ${ }^{67}$ Evidences have been reported that antigen-specific TGF- $\beta$-producing Th 3 cells could maintain peripheral tolerance by promoting the differentiation of antigen-specific Foxp $3^{+}$regulatory cells, and adoptive transfer of myelin oligodendrocyte glycoprotein TCR-Tg $\times$ TgTGF- $\beta$ T cells into wild-type mice induced autoreactive Th3 cells to ameliorate experimental autoimmune encephalomyelitis (EAE) in mice. ${ }^{68}$

\section{REGULATORY B CELLS}

B cells are typically characterized by their capacity to produce antibodies. In addition, they release cytokines and serve as secondary APCs. As with T cells, B cells possess distinct subpopulations that perform pathogenic and regulatory functions, respectively. In 2002, Bhan and Mizoguchi used the term "Bregs" to describe the IL-10-producing B cells, a subpopulation of $B$ cells capable of maintaining immune homeostasis and performing the suppressive function chiefly depending on their secretion of IL-10 (Figure 1) ${ }^{69}$ Regulatory B cells (Bregs) not only regulate the balance of Th1, Th17, and Tregs, and subsequently inhibit inflammation, but also can convert effector T cells into Tr1 cells. Accumulating evidence has demonstrated that Bregs play a regulatory role in certain autoimmune diseases. In the myelin oligodendrocyte glycoprotein-induced EAE mouse model, $B$ cell-deficient mice failed to enter the remission phase, whereas transfer of $\mathrm{IL}-10^{+} \mathrm{B}$ cells could inhibit EAE. ${ }^{69}$ IL-10-producing B cells in mesenteric lymph nodes regulate the progression of chronic colitis of TCR $\alpha$ knockout mice by directly downregulating inflammatory cascades associated with IL- $1 \beta$ production and signal transducer and activator of transcription 3 activation. ${ }^{8}$ The intact gut microbiota could also facilitate IL-10-producing B-cell differentiation in the spleen and mesenteric lymph nodes by increasing IL- $1 \beta$ and IL- 6 production in mice. ${ }^{70}$ In humans, Bregs have been studied primarily in systemic lupus erythematosus and multiple sclerosis, and it was reported that these cells could regulate autoimmune responses. ${ }^{71}$ With regard to IBD, evidence has shown that the frequency of $\mathrm{CD} 19^{\text {high }} \mathrm{CD} 1 \mathrm{~d}^{\text {high }}$ IL-10-producing B cells was reduced in patients with CD, and that this subset of cells could reduce intestinal inflammation in colitis in a Treg-independent manner. ${ }^{72}$ Most recently, IL-33 was found to induce a subset of IL-10-producing B cells $\left(\right.$ Breg $\left.^{\mathrm{IL}-33}\right)$ in mice, and adoptive transfer of these Breg ${ }^{\mathrm{IL}-33}$ into IL-10 ${ }^{-1-}$ mice effectively attenuated mucosal inflammatory responses in the intestines. ${ }^{73}$ Accordingly, evidence has also been generated that certain Breg subsets in performing their suppressive function are dependent on factors other than IL-10. In addition, TGF- $\beta$-producing $B$ cells were observed to have regulatory function in allergic diseases. ${ }^{74} \mathrm{CD} 19^{+} \mathrm{CD} 5^{+}$ Foxp $3^{+}$B cells, a newly discovered type of Bregs, also express Foxp3, but their immunologic function remains unclear. ${ }^{75}$ Furthermore, a novel IL-10-independent immunoregulatory role of B cells has also been found in suppressing autoimmunity, such as EAE, by promoting the expansion of Tregs through GITRL (glucocorticoid-induced TNF ligand). ${ }^{76}$ Moreover, $\mathrm{B}$ cells are found to regulate the immunity and promote 
the recovery of EAE by secreting IL-35. Importantly, CD138 ${ }^{+}$ plasma cells, a subset of activated B cells, produce IL-35 and could prevent intestinal inflammation after salmonella infection. Therefore, these regulatory B cells or plasma cells may provide a novel therapy for IBD. ${ }^{77}$

\section{INNATE LYMPHOID CELLS}

ILCs are recently characterized members of hematopoietic cells of the innate immune system that have been unappreciated for a long period, partly owing to the lack of surface markers distinct from other immune cell lineages. As more and more research work is done in this field, ILCs are emerging to be a brand new star. According to their cytokine profile, ILCs have been classified into three subsets. ${ }^{78}$ The group 1 ILC population, comprising ILC1 and natural killer cells, express T-bet, IFN- $\gamma$, and TNF. The group 2 ILC, similar to Th2 cells, expresses ROR $\alpha$ and GATA3 and could produce Th2 cell-associated cytokines. ILC2 cells consist of natural helper cells, nuocyte, innate helper 2 cells, and multipotent progenitor type 2 cells. In response to IL-25 and IL-33 that are produced by IECs subjected to the infection of helminth, ILC2 could secrete IL-5 and IL-13 to resist the parasite infection. ${ }^{6,79}$ Moreover, studies have shown that lung ILC2 could produce amphiregulin to promote the lung barrier integrity and tissue remodeling after influenza virus-induced damage. ${ }^{80}$ Analogous to Th17 cells, the group 3 ILC population requires the transcription factor ROR $\gamma \mathrm{t}$, and they produce IL-22, IL-17A, and IL-17F. ${ }^{78}$ This group of ILC comprises ILC3 and lymphoid tissue-inducer cells. The lymphoid tissue-inducer cells are essential in secondary lymphoid organ formation during fetal development. A recent report ${ }^{81}$ on dietary effects on the developing immune system has revealed that mouse fetal ILC3 are controlled by both maternal retinoid intake and retinoic acid signaling in a cell-autonomous manner. Although ILCs, particularly ILC1, have been described to participate in the regulation of immune responses in the intestine, the underlying mechanism remains unclear. ROR $\gamma \mathrm{t}^{+}$ILC3 have been recently demonstrated as possibly serving as APCs, owing to the expression of MHC II, but unlike the conventional APCs that do not induce T-cell proliferation. Interestingly, the deletion of MHC II on ROR $\gamma \mathrm{t}^{+}$ILC3 led to commensal bacteriadependent intestinal inflammation, suggesting that ILC3 restrain intestinal $\mathrm{CD} 4{ }^{+} \mathrm{T}$ cell responses to commensal bacteria in an MHC II-dependent manner. ${ }^{82}$ IL-22, important to maintaining intestinal homeostasis, directly induces epithelial defense through the expression of antimicrobial peptides. ${ }^{83}$ As the main source of intestinal IL-22, ROR $\gamma \mathrm{t}^{+}$ILC3 are implicated in the protection and recovery from DSS-mediated epithelial damage and colitis. ${ }^{84}$ Furthermore, ILC3 can also work as potent innate immune effector cells and facilitate resistance to intestinal pathogens (Figure 1) ${ }^{85} \mathrm{IL}$-22-producing ROR $\gamma \mathrm{t}^{+}$ILC3 show the ability to enhance resistance to Gram-negative extracellular bacterial infection at the surfaces of the lungs and intestines. Collectively, identification of the previously unappreciated family of ILCs, which displays various functions in innate and adaptive immunity, might provide new insight into the regulation of intestinal immune responses.

\section{ALTERNATIVELY ACTIVATED MACROPHAGES}

The innate immune cells that are considered to exert the function of bacterial killing, antigen presentation, and proinflammatory cytokine secretion also possess regulatory functions, such as regulatory macrophages and DCs. Macrophages display plasticity in their activation profile under different cytokine conditions. Classically activated macrophages (or M1) induced by IFN- $\gamma$ and microbial products are characterized by microbicidal activity and proinflammatory properties. However, alternatively activated macrophages (AAM; or M2), induced by IL-4 or IL-13, display an anti-inflammatory phenotype. ${ }^{86}$ In humans, AAMs are characterized by a different set of cell surface receptors such as CD163, CD206, DECTIN-1, chemokines (CCL16, CCL17, CCL18, CCL22, CCL24), and chemokine receptors (CCR2, CXCR1, CXCR2). In mice, AAMs also express some specific effector molecules that are induced upon exposure to IL-4/IL-13, such as arginage-1, Fizz1, Ym1, and Ym2. ${ }^{87,88}$ In addition to IL-4 and IL-13, several other cytokines are also found to be involved in AAM induction indirectly through Th2 cells. IL-25 has been found to increase the gene expression of AAMs, and transfer of IL-25-induced AAMs could ameliorate TNBS colitis in mice. ${ }^{89}$ In addition, IL33 could bind to ST2L to increase Th2 cytokine production and AAM induction. ${ }^{90}$ Similar to Th2 cells, ILC2 could also respond to epithelium-derived IL-33 and facilitate AAM polarization by producing IL-13 and IL-5. ${ }^{91}$

AAMs have been found to inhibit the proliferation of $\mathrm{T}$ cells and suppress protozoan infection via cell-to-cell contact or production of regulatory cytokines (e.g., IL-10 and TGF- $\beta$ ) ${ }^{87}$ Therefore, AAMs mainly participate in Th2 cell responses (e.g., chronic obstructive pulmonary disease, asthma) and play a key role in immune regulation (Figure 1).$^{92}$ Accumulating evidence has implied that AAMs could regulate intestinal inflammation. The initial data related to the suppressive function of AAMs in intestinal inflammation come from an interesting phenomenon by which helminth worm infections ameliorate the severity of colitis in mice. ${ }^{93}$ In line with this, a clinical trial has demonstrated the efficacy of the intestinal helminth Trichuris suis in the treatment of active CD patients. ${ }^{94}$ Moreover, injection of AAM significantly ameliorated the severity of dinitrobenzene sulfonic acid-induced colitis in mice by increasing IL-10 production, ${ }^{10}$ indicating that AAMs could maintain the intestinal homeostasis and resist inflammation.

Recent work has shown that active CD patients had low amount of $\mathrm{CD} 68^{+} \mathrm{CD} 206^{+}$macrophages (namely AAMs), whereas colonic biopsies from inactive $\mathrm{CD}$ patients had increased numbers of these cells. ${ }^{10}$ In addition, evidence has shown that anti-TNF therapy could induce $\mathrm{CD} 14^{+}$ monocytes into AAMs in vitro when the $\mathrm{CD} 14^{+}$monocytes were cocultured with $\mathrm{CD} 4{ }^{+} \mathrm{T}$ cells, and further that these AAMs could inhibit the activity of $\mathrm{CD} 4{ }^{+} \mathrm{T}$ cells. ${ }^{95}$ Consistent with this, anti-TNF therapy was found to promote AAM infiltrate in intestinal mucosa of active $\mathrm{CD}$ patients. ${ }^{95}$ The later 
finding certainly implies that AAMs may be involved in the process of IBD, indicating that adoptive transfer of AAMs from patients' blood could be a novel approach in the treatment of IBD. ${ }^{10}$ Our recent data implicated that monocyte could also develop anti-inflammatory properties with producing IL-10 by the induction of insulin-like growth factor-1, and this insulin-like growth factor-1-primed monocyte has the capacity of ameliorating mouse colitis. However, there is no evidence to show that this group of cells possesses the phenotypes like AAMs. ${ }^{96}$

\section{REGULATORY DENDRITIC CELLS}

It has been long established that DCs are one of the most important components of immune system. As a specialized type of APCs, DCs are considered to be the most effective APCs to initiate primary immune responses and be able to link innate and adaptive immunity. After "meeting" foreign intruders, DCs take up antigens, present antigens to T cells, activate them, and further influence the type of immune response.

In recent years, the central role of DCs in inducing inflammatory responses against pathogens and tolerance to commensal microflora in the gut has been established with studies of DC function. There are several DC subsets located at intestinal mucosa that play a crucial role in the maintenance of the gut homeostasis. According to the expression of the surface marker CD103, intestinal DCs can be divided into two predominant subsets: $\mathrm{CD} 11 \mathrm{c}^{\text {high }} \mathrm{CD} 103^{+}$and $\mathrm{CD} 11 \mathrm{c}^{\text {high }}$ CD103 ${ }^{-}$DCs. ${ }^{97}$ Mesenteric lymph node CD11c ${ }^{\text {high }} \mathrm{CD} 103^{-}$ DCs have been reported to increase in murine colitis and exhibit proinflammatory properties. Moreover, Opn-expressing CD11 $c^{\text {high }} \mathrm{CD} 103^{-}$DCs possess the ability to induce Th1 and Th17 cell development. ${ }^{97-99}$ In contrast, one of the major functions of DCs is to help generate Tregs in the gut from naive $\mathrm{T}$ cells. CD11c ${ }^{\text {high }} \mathrm{CD} 103^{+}$DCs have been known for their regulatory functions in the intestine. $\mathrm{CD}_{103}{ }^{+}$DCs derived from the lamina propria of the small intestine have been found to facilitate the differentiation of Tregs in a retinoic acid- and TGF- $\beta$-dependent manner. ${ }^{100}$ However, recently discovered intestinal $\mathrm{CX}_{3} \mathrm{CR}^{\text {high }} \mathrm{CD} 11 \mathrm{~b}^{+} \mathrm{CD} 11 \mathrm{c}^{+}$cells, a subset of $\mathrm{CX} 3 \mathrm{CR}^{+} \mathrm{CD} 11 \mathrm{~b}^{+} \mathrm{CD} 11 \mathrm{c}^{+}$cells that mediate the inflammatory responses, are found to inhibit the proliferation of $\mathrm{CD} 4^{+} \mathrm{T}$ cells and prevent intestinal inflammation. ${ }^{101}$ Furthermore, a type of Tr1-like cells, which exert suppressive functions mainly by secreting IL-10, has been reported to be promoted by $\mathrm{CD} 8 \alpha^{+}$DCs with a $\mathrm{CD} 11 \mathrm{c}^{\text {low }} \mathrm{B} 220^{+}$phenotype, another subpopulation of DCs (Figure 1). ${ }^{102}$

Although the mechanism whereby intestinal DC maintains intestinal homeostasis remains unclear, great progress has been made over the past decade. On one hand, studies have shown that DCs could induce the generation of Tregs directly in the presence of retinoic acid or, as recently reported, CD31conditioned DC could contribute to the development of $\mathrm{CD} 25^{+} \mathrm{Foxp}^{+} \mathrm{T}$ cells dependent on the signaling of coinhibitory receptor CD31. ${ }^{103}$ On the other hand, DCs could combine with other factors to jointly maintain gut homeostasis. When DCs interact with commensal flora, they amplify their immune regulatory functions. Recently, MUC2 secreted by goblet cells has been found to imprint DCs with antiinflammatory abilities. ${ }^{104}$ It augments DC transcription and secretion of TGF- $\beta 1$ and retinaldehyde dehydrogenase (RALDH or ALDH1), an enzyme that converts vitamin A into RA and helps $\mathrm{CD} 103^{+}$DCs to induce Tregs. Moreover, recent studies have highlighted the interaction of DCs and subsets of ILCs in regulating intestinal homeostasis. ${ }^{105,106}$ In the steady state of the gut, after sensing and uptaking microbiota or their products, macrophages secrete IL-1 $\beta$ to stimulate ROR $\gamma \mathrm{t}^{+}$ILC3 to produce colony-stimulating factor 2 that further promotes DCs to maintain Tregs by releasing RA. ${ }^{107}$ Commensal microbiota, tissue-resident mononuclear phagocytes, and ILCs thus form an axis to regulate intestinal homeostasis. $\mathrm{CD}_{11 \mathrm{~b}^{+}}$classical DCs as a source of IL-23 are required for ILC3 activation, that are rendered as a type of immune regulatory cells characterized by producing IL-22, and inhibiting commensal bacteria-specific $\mathrm{CD}_{4}{ }^{+}$T-cell proliferation via MHC II-mediated antigen presentation. ${ }^{106}$

\section{CONCLUSION}

Innate and adaptive immune systems maintain intestinal homeostasis, and regulatory immune cells, including IELs, Tregs, B cells, macrophages, DCs, and ILCs, play a key role in preventing the inappropriate immune responses. However, evidence has been indicated that the existence of an imbalance of proinflammatory and immunosuppressive cells is present in IBD. Although the cytokine milieus are considered to regulate the differentiation of immune cells, the exact mechanism that leads to the dysfunction of regulatory cells in IBD remains unclear. Currently, the main therapies for IBD rely on reducing proinflammatory stimuli, such as anti-TNF treatment. However, restoring the intestinal homeostasis by increasing the frequency and function of immune regulatory cells should be a novel therapeutic approach in the management of IBD. Many studies have demonstrated that the adoptive transfer of these regulatory cells to animal models efficiently alleviates experimental colitis. Therefore, therapy of human IBD with regulatory cells seems warranted.

\section{ACKNOWLEDGMENTS}

We acknowledge funding from the National Natural Science Foundation of China (81270470 and 81470822), NIH DK079918, and DK098370.

\section{DISCLOSURE}

The authors declared no conflict of interest.

c) 2015 Society for Mucosal Immunology

\section{REFERENCES}

1. Garrett, W.S., Gordon, J.I. \& Glimcher, L.H. Homeostasis and inflammation in the intestine. Cell 140, 859-870 (2010).

2. Bernstein, C.N., Wajda, A. \& Blanchard, J.F. The clustering of other chronic inflammatory diseases in inflammatory bowel disease: a population-based study. Gastroenterology 129, 827-836 (2005).

3. Wu, W. et al. miR-10a inhibits dendritic cell activation and Th1/Th17 cell immune responses in IBD. Gut; advance online publication, 3 October 2014; doi:10.1136/gutjnl-2014-307980 (2014).

4. Liu, Z. et al. Interleukin (IL)-23 suppresses IL-10 in inflammatory bowel disease. J. Biol. Chem. 287, 3591-3597 (2012). 
5. Liu, Z. et al. The increased expression of IL-23 in inflammatory bowel disease promotes intraepithelial and lamina propria lymphocyte inflammatory responses and cytotoxicity. J. Leukoc. Biol. 89, 597-606 (2011).

6. Spits, H. \& Cupedo, T. Innate lymphoid cells: emerging insights in development, lineage relationships, and function. Annu. Rev. Immunol. 30, 647-675 (2012).

7. Wing, K. \& Sakaguchi, S. Regulatory Tcells exert checks and balances on self tolerance and autoimmunity. Nat. Immunol. 11, 7-13 (2010).

8. Mizoguchi, A., Mizoguchi, E., Takedatsu, H., Blumberg, R.S. \& Bhan, A.K. Chronic intestinal inflammatory condition generates IL-10-producing regulatory B cell subset characterized by CD1d upregulation. Immunity 16, 219-230 (2002).

9. Matteoli, G. et al. Gut CD103 + dendritic cells express indoleamine 2, 3-dioxygenase which influences $T$ regulatory/T effector cell balance and oral tolerance induction. Gut 59, 595-604 (2010).

10. Hunter, M.M. et al. In vitro-derived alternatively activated macrophages reduce colonic inflammation in mice. Gastroenterology 138, 1395-1405 (2010).

11. Cao, A.T., Yao, S., Gong, B., Elson, C.O. \& Cong, Y. Th17 cells upregulate polymeric Ig receptor and intestinal IgA and contribute to intestinal homeostasis. J. Immunol. 189, 4666-4673 (2012).

12. Cao, A.T. et al. Interleukin (IL)-21 promotes intestinal IgA response to microbiota. Mucosal Immunol; advance online publication, 14 January 2015; doi:10.1038/mi.2014.134 (2015).

13. Coccia, M. et al. IL-1beta mediates chronic intestinal inflammation by promoting the accumulation of IL-17A secreting innate lymphoid cells and CD4(+) Th17 cells. J. Exp. Med. 209, 1595-1609 (2012).

14. Maxwell, J.R. et al. IL-18 bridges innate and adaptive immunity through IFN-gamma and the CD134 pathway. J. Immunol. 177, 234-245 (2006).

15. Dupaul-Chicoine, J. et al. Control of intestinal homeostasis, colitis, and colitis-associated colorectal cancer by the inflammatory caspases. Immunity 32, 367-378 (2010).

16. Grobeta, P., Doser, K., Falk, W., Obermeier, F. \& Hofmann, C. IL-33 attenuates development and perpetuation of chronic intestinal inflammation. Inflamm. Bowel Dis. 18, 1900-1909 (2012).

17. Schiering, C. et al. The alarmin IL-33 promotes regulatory T-cell function in the intestine. Nature 513, 564-568 (2014).

18. McNamee, E.N. et al. Interleukin 37 expression protects mice wfrom colitis. Proc. Natl. Acad. Sci. USA 108, 16711-16716 (2011).

19. Liu, H. et al. ERK differentially regulates Th17- and Treg-cell development and contributes to the pathogenesis of colitis. Eur. J. Immunol. 43, 17161726 (2013).

20. Suzuki, A. et al. CIS3/SOCS3/SSI3 plays a negative regulatory role in STAT3 activation and intestinal inflammation. J. Exp. Med. 193, 471-481 (2001).

21. Sander, L.E. et al. Gp130 signaling promotes development of acute experimental colitis by facilitating early neutrophil/macrophage recruitment and activation. J. Immunol. 181, 3586-3594 (2008).

22. Beagley, K.W. et al. Interleukins and IgA synthesis. Human and murine interleukin 6 induce high rate $\operatorname{lgA}$ secretion in IgA-committed B cells. J. Exp. Med. 169, 2133-2148 (1989).

23. Su, J. et al. IL-25 downregulates Th1/Th17 immune response in an IL-10dependent manner in inflammatory bowel disease. Inflamm. Bowel Dis. 19, 720-728 (2013).

24. Van der Sluis, M. etal. Muc2-deficient mice spontaneously develop colitis, indicating that MUC2 is critical for colonic protection. Gastroenterology 131, 117-129 (2006).

25. Vereecke, L. et al. A20 controls intestinal homeostasis through cellspecific activities. Nat. Commun. 5, 5103 (2014).

26. Vereecke, L. et al. Enterocyte-specific A20 deficiency sensitizes to tumor necrosis factor-induced toxicity and experimental colitis. J. Exp. Med. 207, 1513-1523 (2010).

27. Cheroutre, H., Lambolez, F. \& Mucida, D. The light and dark sides of intestinal intraepithelial lymphocytes. Nat. Rev. Immunol. 11, 445-456 (2011).

28. Cheroutre, H. \& Lambolez, F. Doubting the TCR coreceptor function of CD8alphaalpha. Immunity 28, 149-159 (2008).

29. Simpson, S.J. et al. Expression of pro-inflammatory cytokines by TCR alpha beta + and TCR gamma delta + Tcells in an experimental model of colitis. Eur. J. Immunol. 27, 17-25 (1997).
30. Edelblum, K.L. et al. Dynamic migration of gammadelta intraepithelial lymphocytes requires occludin. Proc. Natl. Acad. Sci. USA 109, 7097-7102 (2012).

31. Edelblum, K.L. et al. $\gamma \delta$ intraepithelial lymphocyte migration limits transepithelial pathogen invasion and systemic disease in mice. Gastroenterology, piiS0016-5085, 00302-00309 (2015).

32. Meehan, T.F. et al. Protection against colitis by CD100-dependent modulation of intraepithelial gammadelta T lymphocyte function. Mucosal Immunol. 7, 134-142 (2014).

33. Chen, Y., Chou, K., Fuchs, E., Havran, W.L. \& Boismenu, R. Protection of the intestinal mucosa by intraepithelial gamma delta Tcells. Proc. Natl. Acad. Sci. USA 99, 14338-14343 (2002).

34. Kober, O.l. et al. $\gamma \delta$ T-cell-deficient mice show alterations in mucin expression, glycosylation, and goblet cells but maintain an intact mucus layer. Am. J. Physiol. Gastrointest. Liver Physiol. 306, G582-G593 (2014).

35. Inagaki-Ohara, K. et al. Mucosal T cells bearing TCRgammadelta play a protective role in intestinal inflammation. J. Immunol. 173, 1390-1398 (2004).

36. Kuhl, A.A. et al. Human peripheral gammadelta Tcells possess regulatory potential. Immunology 128, 580-588 (2009).

37. Denning, T.L. et al. Mouse TCRalphabeta + CD8alphaalpha intraepithelial lymphocytes express genes that down-regulate their antigen reactivity and suppress immune responses. J. Immunol. 178, 4230-4239 (2007).

38. Poussier, P., Ning, T., Banerjee, D. \& Julius, M. A unique subset of selfspecific intraintestinal T cells maintains gut integrity. J. Exp. Med. 195, 1491-1497 (2002).

39. Abbas, A.K. et al. Regulatory T cells: recommendations to simplify the nomenclature. Nat. Immunol. 14, 307-308 (2013).

40. Fontenot, J.D., Gavin, M.A. \& Rudensky, A.Y. Foxp3 programs the development and function of CD4 $+\mathrm{CD} 25+$ regulatory T cells. Nat. Immunol. 4, 330-336 (2003).

41. Hori, S., Nomura, T. \& Sakaguchi, S. Control of regulatory T cell development by the transcription factor Foxp3. Science 299, 1057-1061 (2003).

42. Bennett, C.L. \& Ochs, H.D. IPEX is a unique X-linked syndrome characterized by immune dysfunction, polyendocrinopathy, enteropathy, and a variety of autoimmune phenomena. Curr. Opin. Pediatr. 13, 533538 (2001).

43. Fantini, M.C. et al. Cutting edge: TGF-beta induces a regulatory phenotype in CD4+CD25- T cells through Foxp3 induction and down-regulation of Smad7. J. Immunol. 172, 5149-5153 (2004).

44. Shevach, E.M. Mechanisms of foxp3 + T regulatory cell-mediated suppression. Immunity 30, 636-645 (2009).

45. Read, S. et al. Blockade of CTLA-4 on CD4 + CD25 + regulatory T cells abrogates their function in vivo. J. Immunol. 177, 4376-4383 (2006).

46. Maul, J. et al. Peripheral and intestinal regulatory CD4 + CD25(high) T cells in inflammatory bowel disease. Gastroenterology 128, 1868-1878 (2005).

47. Ehrenstein, M.R. et al. Compromised function of regulatory T cells in rheumatoid arthritis and reversal by anti-TNFalpha therapy. J. Exp. Med. 200, 277-285 (2004).

48. Ueno, A. et al. Increased prevalence of circulating novel IL-17 secreting Foxp3 expressing CD4 + T cells and defective suppressive function of circulating Foxp3 + regulatory cells support plasticity between Th17 and regulatory T cells in inflammatory bowel disease patients. Inflamm. Bowel Dis. 19, 2522-2534 (2013).

49. Wu, W. et al. Prolactin mediates psychological stress-induced dysfunction of regulatory Tcells to facilitate intestinal inflammation. Gut $63,1883-$ 1892 (2014).

50. Veltkamp, C. et al. Apoptosis of regulatory T lymphocytes is increased in chronic inflammatory bowel disease and reversed by anti-TNF $\alpha$ treatment. Gut 60, 1345-1353 (2011).

51. Boschetti, G. et al. Therapy with anti-TNFalpha antibody enhances number and function of Foxp3 $(+)$ regulatory Tcells in inflammatory bowel diseases. Inflamm. Bowel Dis. 17, 160-170 (2011).

52. Liu, Z., Cao, A.T. \& Cong, Y. Microbiota regulation of inflammatory bowel disease and colorectal cancer. Semin. Cancer Biol. 23, 543-552 (2013).

53. Hayashi, A. et al. A single strain of Clostridium butyricum induces intestinal IL-10-producing macrophages to suppress acute experimental colitis in mice. Cell Host Microbe 13, 711-722 (2013). 
54. Ivanov, I.I. et al. Induction of intestinal Th17 cells by segmented filamentous bacteria. Cell 139, 485-498 (2009).

55. Round, J.L. \& Mazmanian, S.K. Inducible Foxp3 + regulatory T-cell development by a commensal bacterium of the intestinal microbiota. Proc. Natl. Acad. Sci. USA 107, 12204-12209 (2010).

56. Izcue, A. et al. Interleukin-23 restrains regulatory $T$ cell activity to drive $T$ cell-dependent colitis. Immunity 28, 559-570 (2008).

57. Korn, T. et al. IL-21 initiates an alternative pathway to induce proinflammatory TH17 cells. Nature 448, 484-487 (2007).

58. Atarashi, K. et al. Induction of colonic regulatory $\mathrm{T}$ cells by indigenous Clostridium species. Science 331, 337-341 (2011).

59. Furusawa, Y. et al. Commensal microbe-derived butyrate induces the differentiation of colonic regulatory T cells. Nature 504, 446-450 (2013).

60. Chang, P.V., Hao, L., Offermanns, S. \& Medzhitov, R. The microbial metabolite butyrate regulates intestinal macrophage function via histone deacetylase inhibition. Proc. Natl. Acad. Sci. USA 111, 2247-2252 (2014).

61. Singh, N. et al. Activation of Gpr109a, receptor for niacin and the commensal metabolite butyrate, suppresses colonic inflammation and carcinogenesis. Immunity 40, 128-139 (2014).

62. Roncarolo, M.G. et al. Interleukin-10-secreting type 1 regulatory T cells in rodents and humans. Immunol. Rev. 212, 28-50 (2006).

63. Groux, H. et al. A CD4 ${ }^{+}$T-cell subset inhibits antigen-specific T-cell responses and prevents colitis. Nature 389, 737-742 (1997).

64. Cong, Y., Weaver, C.T., Lazenby, A. \& Elson, C.O. Bacterial-reactive T regulatory cells inhibit pathogenic immune responses to the enteric flora. J. Immunol. 169, 6112-6119 (2002).

65. Pestka, S. et al. Interleukin-10 and related cytokines and receptors. Annu. Rev. Immunol. 22, 929-979 (2004).

66. Desreumaux, P. et al. Safety and efficacy of antigen-specific regulatory T-cell therapy for patients with refractory Crohn's disease. Gastroenterology 143, 1207-1217 e1202 (2012).

67. Chen, Y., Kuchroo, V.K., Inobe, J., Hafler, D.A. \& Weiner, H.L. Regulatory T cell clones induced by oral tolerance: suppression of autoimmune encephalomyelitis. Science 265, 1237-1240 (1994).

68. Carrier, Y., Yuan, J., Kuchroo, V.K. \& Weiner, H.L. Th3 cells in peripheral tolerance. I. Induction of Foxp3-positive regulatory T cells by Th3 cells derived from TGF-beta Tcell-transgenic mice. J. Immunol. 178, 179-185 (2007).

69. Fillatreau, S., Sweenie, C.H., McGeachy, M.J., Gray, D. \& Anderton, S.M. B cells regulate autoimmunity by provision of IL-10. Nat. Immunol. 3, 944950 (2002)

70. Rosser, E.C. et al. Regulatory B cells are induced by gut microbiota-driven interleukin-1 beta and interleukin-6 production. Nat. Med. 20, 1334-1339 (2014).

71. Duddy, M. et al. Distinct effector cytokine profiles of memory and naive human B cell subsets and implication in multiple sclerosis. J. Immunol. 178, 6092-6099 (2007).

72. Oka, A. et al. Role of regulatory B cells in chronic intestinal inflammation: association with pathogenesis of Crohn's disease. Inflamm. Bowel Dis. 20, 315-328 (2014).

73. Sattler, S. et al. IL-10-producing regulatory B cells induced by IL-33 (Breg(IL-33)) effectively attenuate mucosal inflammatory responses in the gut. J. Autoimmun. 50, 107-122 (2014).

74. Lee, J.H. et al. Allergen-specific transforming growth factor- $\beta$-producing CD19 $(+)$ CD5 $(+)$ regulatory $B$-cell $(\mathrm{Br} 3)$ responses in human late eczematous allergic reactions to cow's milk. J. Interferon Cytokine Res. 31, 441-449 (2011).

75. Noh, J., Choi, W.S., Noh, G. \& Lee, J.H. Presence of Foxp3-expressing CD19 $(+)$ CD5 $(+)$ B cells in human peripheral blood mononuclear cells: human CD19 $(+)$ CD5 $(+)$ Foxp3 $(+)$ regulatory B cell (Breg). Immune. Netw. 10, 247-249 (2010).

76. Ray, A., Basu, S., Williams, C.B., Salzman, N.H. \& Dittel, B.N. A novel IL10-independent regulatory role for B cells in suppressing autoimmunity by maintenance of regulatory Tcells via GITR ligand. J. Immunol. 188, 31883198 (2012).

77. Shen, P. et al. IL-35-producing B cells are critical regulators of immunity during autoimmune and infectious diseases. Nature 507, 366-370 (2014).
78. Walker, J.A., Barlow, J.L. \& McKenzie, A.N. Innate lymphoid cells-how did we miss them? Nat. Rev. Immunol. 13, 75-87 (2013).

79. Tait Wojno, E.D. \& Artis, D. Innate lymphoid cells: balancing immunity, inflammation, and tissue repair in the intestine. Cell Host Microbe 12, 445-457 (2012).

80. Monticelli, L.A. et al. Innate lymphoid cells promote lung-tissue homeostasis after infection with influenza virus. Nat. Immunol. 12, 1045-1054 (2011).

81. van de Pavert, S.A. et al. Maternal retinoids control type 3 innate lymphoid cells and set the offspring immunity. Nature 508, 123-127 (2014).

82. Hepworth, M.R. et al. Innate lymphoid cells regulate CD4+ T-cell responses to intestinal commensal bacteria. Nature 498, 113-117 (2013).

83. Wolk, K. et al. IL-22 increases the innate immunity of tissues. Immunity $\mathbf{2 1}$, 241-254 (2004)

84. Sawa, S. et al. RORgammat + innate lymphoid cells regulate intestinal homeostasis by integrating negative signals from the symbiotic microbiota. Nat. Immunol. 12, 320-326 (2011).

85. Spits, H. \& Di Santo, J.P. The expanding family of innate lymphoid cells: regulators and effectors of immunity and tissue remodeling. Nat. Immunol. 12, 21-27 (2011).

86. Mosser, D.M. \& Edwards, J.P. Exploring the full spectrum of macrophage activation. Nat. Rev. Immunol. 8, 958-969 (2008).

87. Gordon, S. \& Martinez, F.O. Alternative activation of macrophages: mechanism and functions. Immunity 32, 593-604 (2010).

88. Nair, M.G. et al. Alternatively activated macrophage-derived RELM- $\alpha$ is a negative regulator of type 2 inflammation in the lung. J. Exp. Med. 206, 937-952 (2009).

89. Rizzo, A. et al. Inhibition of colitis by IL-25 associates with induction of alternatively activated macrophages. Inflamm. Bowel Dis. 18, 449-459 (2012).

90. Kurowska-Stolarska, M. et al. IL-33 amplifies the polarization of alternatively activated macrophages that contribute to airway inflammation. J. Immunol. 183, 6469-6477 (2009).

91. Besnard, A.G. et al. IL-33-mediated protection against experimental cerebral malaria is linked to induction of type 2 innate lymphoid cells, M2 macrophages and regulatory Tcells. PLoS Pathog. 11, e1004607 (2015).

92. Byers, D.E. \& Holtzman, M.J. Alternatively activated macrophages and airway disease. Chest 140, 768-774 (2011).

93. Smith, P. et al. Infection with a helminth parasite prevents experimental colitis via a macrophage-mediated mechanism. J. Immunol. 178, 45574566 (2007).

94. Summers, R., Elliott, D., Urban, J., Thompson, R. \& Weinstock, J. Trichuris suis therapy in Crohn's disease. Gut 54, 87-90 (2005).

95. Vos, A.C. et al. Anti-tumor necrosis factor-alpha antibodies induce regulatory macrophages in an Fc region-dependent manner. Gastroenterology 140, 221-230 (2011).

96. Ge, R.T. et al. Insulin-like growth factor-1 endues monocytes with immune suppressive ability to inhibit inflammation in the intestine. Sci. Rep. 5, 7735 (2015)

97. Varol, C. et al. Intestinal lamina propria dendritic cell subsets have different origin and functions. Immunity 31, 502-512 (2009).

98. Niess, J.H. \& Adler, G. Enteric flora expands gut lamina propria CX3CR1 + dendritic cells supporting inflammatory immune responses under normal and inflammatory conditions. J. Immunol. 184, 2026-2037 (2010).

99. Atarashi, $\mathrm{K}$. et al. ATP drives lamina propria TH17 cell differentiation. Nature 455, 808-812 (2008).

100. Coombes, J.L. et al. A functionally specialized population of mucosal CD103 + DCs induces Foxp3 + regulatory T cells via a TGF-beta and retinoic acid-dependent mechanism. J. Exp. Med. 204, 1757-1764 (2007)

101. Kayama, H. et al. Intestinal CX3C chemokine receptor 1(high) (CX3CR1(high)) myeloid cells prevent T-cell-dependent colitis. Proc. Natl. Acad. Sci. USA 109, 5010-5015 (2012).

102. Bilsborough, J. \& Viney, J.L. In vivo enhancement of dendritic cell function. Ann. NY Acad. Sci. 1029, 83-87 (2004).

103. Clement, M. et al. CD31 is a key coinhibitory receptor in the development of immunogenic dendritic cells. Proc. Natl. Acad. Sci. USA 111, E1101E1110 (2014). 


\section{REVIEW}

104. Shan, M. et al. Mucus enhances gut homeostasis and oral tolerance by delivering immunoregulatory signals. Science 342, 447-453 (2013).

105. Kinnebrew, M.A. et al. Interleukin 23 production by intestinal CD103 $(+) \mathrm{CD} 11 \mathrm{~b}(+)$ dendritic cells in response to bacterial flagellin enhances mucosal innate immune defense. Immunity 36, 276-287 (2012).
106. Satpathy, A.T. et al. Notch2-dependent classical dendritic cells orchestrate intestinal immunity to attaching-and-effacing bacterial pathogens. Nat. Immunol. 14, 937-948 (2013).

107. Mortha, A. et al. Microbiota-dependent crosstalk between macrophages and ILC3 promotes intestinal homeostasis. Science 343, 1249288 (2014). 